\title{
Interacting Turbulent Plumes in a Naturally Ventilated Enclosure
}

\author{
P. F. Linden ${ }^{1}$ and N. B. Kaye ${ }^{2}$ \\ ${ }^{1}$ Department of Mechanical and Aerospace Engineering, University of California, San Diego, \\ 9500 Gilman Drive, La Jolla, CA 92093-0411, USA.
}

${ }^{2}$ Department of Civil and Environmental Engineering, Imperial College of Science, Technology and Medicine, Imperial College Road, London, SW7 2BU, UK

\begin{abstract}
The interaction of turbulent plumes is examined in the context of building ventilation flows. Recent models for natural ventilation have been based on simplified treatment of the heat sources in a ventilated enclosure. These models treat buoyancy sources as plumes and assume that they do not interact. To improve the applicability of these models to buildings we examine the effects of plume-plume interaction on the ventilation flow. We present experimental results for the flow in a ventilated enclosure where two plumes in close proximity to each other coalesce to form a single plume. We also examine the case of two plumes of opposing buoyancy flux colliding in a ventilated enclosure. Theoretical models for the effect of this interaction on the standard ventilation model of Linden, Lane-Serff and Smeed (1990) are also discussed.
\end{abstract}

Key words: natural ventilation, interacting plumes, buoyancy driven turbulence.

\section{Introduction}

In recent years much attention has been paid to developing simplified models for natural ventilation. These models consider the ventilation of an enclosure with openings to the exterior driven by interior heat sources. These heat sources are either represented as isolated sources producing noninteracting plumes (Linden et al 1990) or as a uniform distribution of heat over the floor (Gladstone et al 2001). In the latter case the temperature within the enclosure is uniform with height, while a two-layer stratification forms for equal-strength sources. The steady-state ventilation flows produced by multiple non-interacting plumes of different strengths produce complex internal stratifications (Cooper et al 1996, Linden et al 1996), the properties of which are determined, like the ventilation rate, by entrainment of air into the rising plumes. Interaction between plumes will result in a reduction in net entrainment from the environment as the plumes partially entrain from each other, reducing their net flow rate. This change in entrainment will alter building ventilation rates and the internal temperature stratification.

In Section 2 we review building ventilation models for single and multiple plumes in the absence of external driving forces such as wind and distributed solar gains. We present theory and experimental results for the case of two co-flowing, coalescing plumes in Section 3 and for colliding plumes in Section 4. Broader implications of this work and conclusions are given in Section 5.

\section{Review of Ventilation Theory}

We consider a plume in an enclosure where the fluid in the enclosure can be exchanged with the ambient fluid outside the enclosure, but the fluid in the plume cannot (Figure 1).

When a heat source is introduced into an empty enclosure (where the internal ambient density is the same as the external) the plume above the heat source creates a warm layer at the ceiling. An interface forms at the base of this layer and starts to descend toward the plume source. This creates a stable stratification in the enclosure. The presence of the warm buoyant layer produces a pressure difference between the enclosure and the exterior, which drives a flow through the enclosure. Ambient fluid enters at the bottom of the enclosure, is entrained into the plume, and leaves through the upper opening. Over time the density interface reaches a steady height with the flow rate through the enclosure equal to the flow rate in the plume at the interface height. In the steady state, the buoyancy flux out of the enclosure is equal to the 


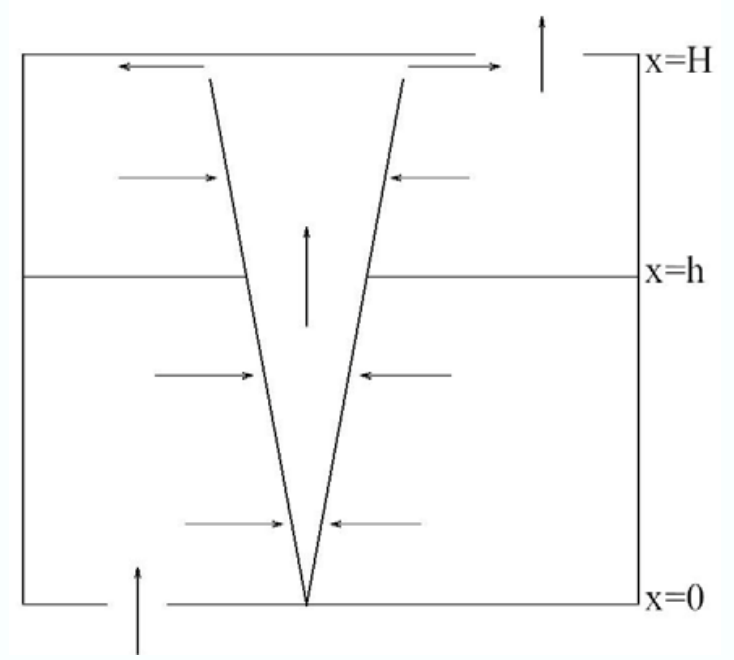

Figure 1. A ventilated enclosure showing the flow through the enclosure driven by the buoyant plume, and the steady state interface.

buoyancy flux of the heat source. The buoyancy of the upper layer must, therefore, equal the buoyancy of the plume at the interface. This balance of buoyancy and volume fluxes leads to the following expression for the interface height $\zeta=\mathrm{h} / \mathrm{H}$ :

$\frac{A^{*}}{H^{2}}=C^{3 / 2} \bigcap{\frac{\zeta^{5}}{1-\zeta}}^{1 / 2}$

where $\mathrm{A}^{*}$ is an effective area for the inlet and outlet, defined as:

$$
A^{*}=\frac{a_{1} a_{2}}{\left(\frac{1}{2} C_{e} C_{d}\left(\oiint_{e} a_{1}^{2}+C_{d} a_{2}^{2}\right)^{1 / 2}\right.} .
$$

$\mathrm{C}$ is given by:

$C=\frac{6}{5} \alpha\left(\frac{18}{5} \alpha\right)^{1 / 3} \pi^{2 / 3}$,

$a_{1}$ is the outlet area, $a_{2}$ is the inlet area, and $C_{e}$ and $\mathrm{C}_{\mathrm{d}}$ are discharge coefficients (for a more detailed discussion of discharge coefficients and effective areas see Cooper et al (1996)). $\alpha=0.083$ is the entrainment coefficient for a point source plume. The upper layer buoyancy is given by:

$g^{\prime}=\frac{\hat{F}^{2 / 3}}{h^{5 / 3} C}$ where $\hat{F}$ is the buoyancy flux of the plume. Note that the height of the interface is independent of the plume buoyancy flux. However, the buoyancy of the upper layer does increase with increasing buoyancy flux.

If there are $n$ equal non-interacting plumes, since they share the opening areas, Equation 1 becomes:

$$
\frac{1}{n} \frac{A^{*}}{H^{2}}=C^{3 / 2} \bigcap{\frac{\zeta_{n}^{5}}{1-\zeta_{n}}}^{1 / 2}
$$

where $\zeta_{n}$ is the interface height for the case of $n$ non-interacting plumes (Linden et al 1990). Two non-interacting sources of buoyancy of differing strengths create a three-layer system. In this case the interface heights are functions of the effective areas and the ratio of the buoyancy fluxes $\psi=\hat{F}_{2} / \hat{F}_{1}$ (Cooper et al 1996). This ratio $\psi$ is defined such that the stronger plume is taken to be $\hat{F}_{1}$ so that $0<\psi<1$. When the weaker plume reaches the upper layer it spreads out, forming an intermediate layer. This plume also hits the upper interface and entrains fluid back down into the middle layer (Figure 2). Multiple sources of buoyancy located on the floor produce a multi-layered system (Linden et al 1996).

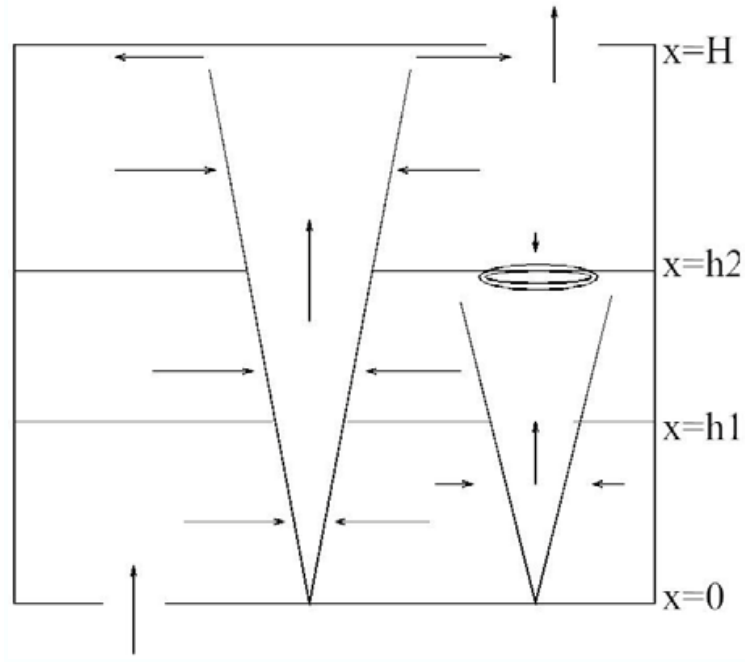

Figure 2. A ventilated enclosure with two sources of buoyancy, showing the three layers and entrainment across the top interface due to the weaker plume impinging on it (with mixing and entrainment shown by the circles above the weaker plume). 


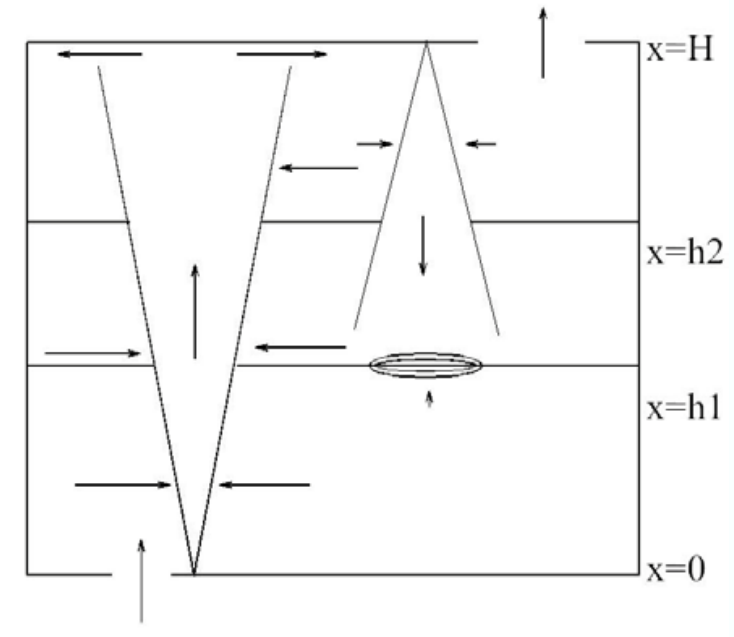

Figure 3. A ventilated enclosure containing two sources of buoyancy of opposite sign. The schematic shows the three layers and the entrainment across the lower layer due to the weaker plume impinging on it.

When the heat sources have different signs, opposing plumes may occur, for example, when air conditioning vents are located in the ceiling of a room with heat sources near the floor. Again a three layer stratification develops for most cases (Cooper et al 1996). For low values of $|\psi|$ the stronger plume (positively buoyant by convention) produces a buoyant upper layer, and the weaker, negatively buoyant plume falls through that layer. This plume entrains warm upper layer fluid and can become positively buoyant, so that the plume has positive buoyancy but negative momentum. This turbulent fountain then falls a distance dependent on its momentum, before rising back up to form a middle layer. In the steady state the three layers persist, with the turbulent fountain entraining fluid from the lower ambient layer into the middle layer (Figure 3). Again the layer heights are dependent only on the geometry of the problem and on $\psi$.

In the case of larger $|\psi|$ the weaker plume can break through the upper layer and still remain negatively buoyant. In this case the lower layer becomes negatively buoyant, producing an exchange flow through the lower vents, which has not been investigated analytically. When $|\psi|=1$ there is no net heat input, and there is no net flow through the enclosure.

These analyses assume that there is no plume-plume interaction. Any interaction will act to reduce entrainment into the plumes, and reduce the flow rates in the plumes. This reduction in flow rate will consequently reduce the ventilation flow rate through the enclosure. It is, therefore, important to understand the extent of plume-plume interaction in these flows.

\section{Co-flowing Coalescing Plumes}

Co-flowing turbulent plumes entrain fluid from the region between them and eventually coalesce. The plumes can be considered as separate up to a coalescence height $z_{m}$ that is a function of their horizontal source separation $\chi_{0}$ and the ratio of their buoyancy fluxes $\psi$. For equal plumes $z_{m}=4.8 \chi_{0}$ (Kaye and Linden 2004) and, for unequal plumes, $z_{m}$ decreases as $\psi$ decreases. Above this height the flow behaves as though it were a single plume, and for equal plumes the combined plume has buoyancy flux $2 \hat{F}$ and originates from a virtual origin $z_{v}=1.38 \chi_{0}$ below the original plume sources (Figure 4).

As reviewed in Section 2 the non-interacting ventilation model predicts a two-layer stratification (Linden et al 1990). The coalescence of two turbulent plumes implies that above $Z_{c}$ the total volume flux is reduced compared to that of the noninteracting case. Thus the interface height will be higher, and the flow driven through the enclosure will be reduced.

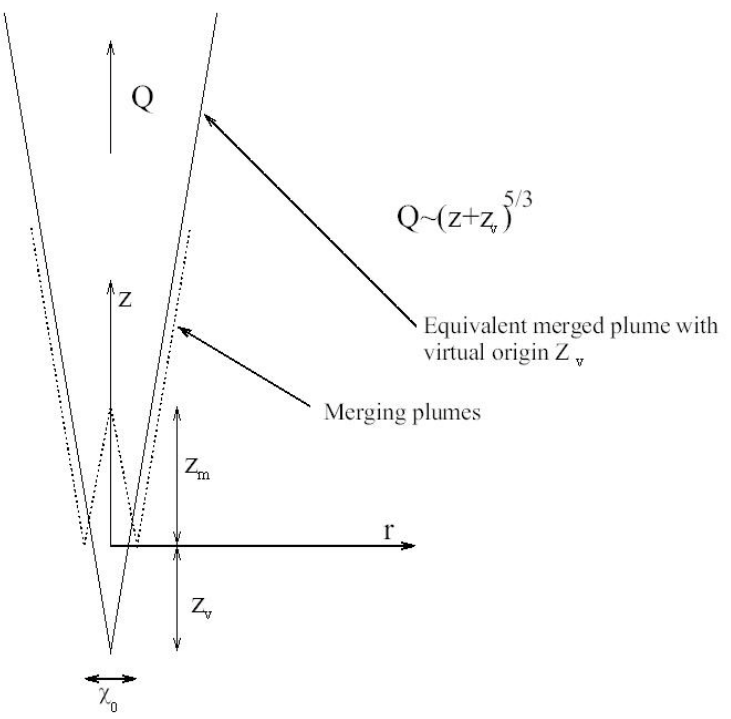

Figure 4. Schematic of two merging turbulent plumes showing the resulting single plume with origin offset. 


\subsection{Equal Plumes}

In a ventilated enclosure with two equal but noninteracting plumes, each of buoyancy flux $\hat{F}$, the steady state buoyancy profile is a two layer stratification with the interface height given by Equation 5 with $n=2$ :

$$
\frac{1}{2} \frac{A^{*}}{H^{2}}=C^{3 / 2}\left(\frac{\zeta_{2}^{5}}{1-\zeta_{2}}\right)^{1 / 2}
$$

If two plumes merge at their source, there is a single plume with twice the buoyancy flux, and the interface height and upper-layer buoyancy are then given by:

$$
\frac{A^{*}}{H^{2}}=C^{3 / 2}\left(\frac{\zeta_{2}^{5}}{1-\zeta_{2}}\right)^{1 / 2}
$$

and
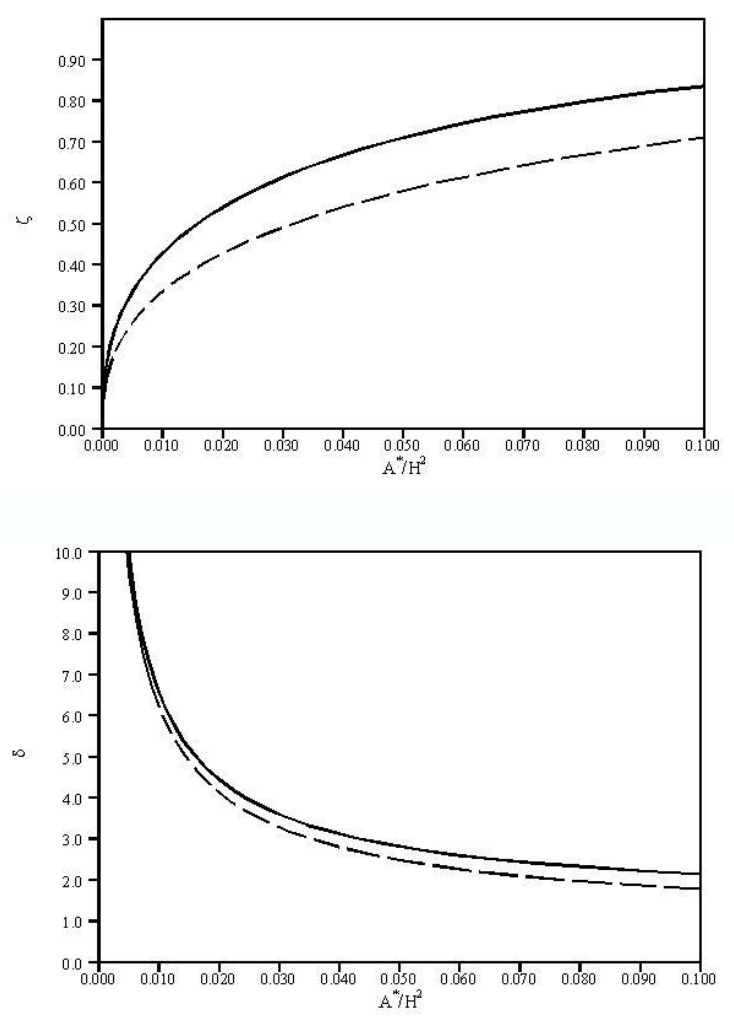

Figure 5. Interface height and upper layer buoyancy as a function of $A^{*} / H^{2}$ for a single plume and two equal plumes. The solid line is the single plume solution. $g^{\prime}=\frac{(2 \hat{F})^{2 / 3}}{h^{5 / 3} C}$

respectively.

The interface height and upper-layer buoyancy for two equal plumes and a single plume of twice the buoyancy flux, which represent the upper and lower bounds on the problem of two equal merging turbulent plumes, are shown in Figure 5. The buoyancy is scaled on the buoyancy of a plume of buoyancy flux $\hat{F}$ at a height $H$ above its source. That is:

$\delta \xi=\frac{g^{\prime}}{C H^{-\frac{5}{3}} \hat{F}^{\frac{2}{3}}} \quad-\frac{5}{3}$

Although the difference in the buoyancy of the upper layer is small, the change in interface height is significant. For example at $A^{*} / H^{2}=0.05$,
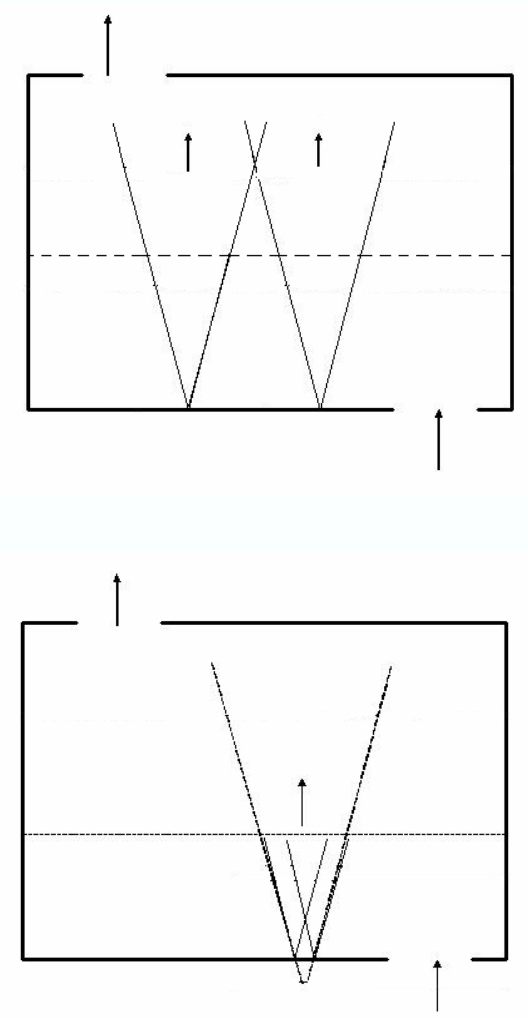

Figure 6. Schematic showing two equal plumes merging above (top) and below (bottom) the buoyancy interface in a ventilated enclosure. Also shown (bottom) is the equivalent single plume formed by the merged plumes. 
$\zeta_{1}=0.71$ and $\zeta_{2}=0.58$, where the subscript refers to the number of non-interacting plumes present. In a typical office with a $2.5 \mathrm{~m}$ ceiling, that represents a difference in interface height of about $0.3 \mathrm{~m}$.

There are two possible scenarios depending on the coalescence height. Either the plumes merge below the interface, in which case the single plume solution can be used with an offset for the virtual origin, or they merge above the interface, in which case the two non-interacting plume result is used (Figure 6).

For a given $\mathrm{A}^{*} / \mathrm{H}^{2}$ the single plume and double plume interface heights can be calculated from Equation 5 as $\zeta_{1}$ and $\zeta_{2}$, respectively. Provided the plumes merge below the two-plume interface height, that is $\zeta_{2}>4.8 \sigma_{0}$, the new interface height can be written as $\zeta^{\prime}=\zeta_{1}+\varepsilon$, where $\varepsilon$ is a correction to the single plume solution due to the apparent increase in height caused by the virtual origin of the merged plume. From Equation 1:

$\frac{A^{*}}{\left(H+z_{v}\right)^{2}}=C^{3 / 2}\left(\frac{\left(z^{\prime}+z_{v}\right)^{5}\left(H+z_{v}\right)^{-5}}{1-\frac{z^{\prime}+z_{v}}{H+z_{v}}}\right)^{1 / 2}$

which can be re-written as:

$\frac{A^{*}}{H^{2}}=C^{3 / 2}\left(\frac{\left(\zeta_{1}+\varepsilon+\zeta_{v}\right)^{5}}{1-\zeta_{1}-\varepsilon}\right)^{1 / 2}$

For small perturbations to the interface height:

$\varepsilon \approx-\zeta_{v}\left(\frac{5 \zeta_{1}^{4}}{\left({\frac{A^{*}}{H^{2} C^{3 / 2}}}^{2}+5 \zeta_{1}^{4}\right.}\right)$

Since the location $\zeta_{v}$ of the virtual origin of the merged plume is linearly related to the initial plume separation $\sigma_{0}$, the interface height will vary approximately linearly with separation from the single plume at zero separation to the solution for two-separate plumes. Substituting Equation 7 into Equation 12 gives:

$\varepsilon \approx-\zeta_{v}\left(\frac{1-\zeta_{1}}{1-\frac{4}{5} \zeta_{1}}\right)$
For a given value of $\zeta_{v}$ it is possible to see how the interface height varies with vent area. When the opening area is small $\zeta$ will also be small and, therefore, $\varepsilon \approx-\zeta_{v}$. However for larger vent areas the value of $\zeta$ will get closer to 1 and $\varepsilon$ will get smaller. That is, the difference between the singleplume solution and the solution for two merging plumes will be less for larger opening areas.

\subsection{Unequal Plumes}

As discussed in Section 2, two non-interacting unequal plumes produce a three layer stratification. The effect of plume-plume interaction on this three layer stratification is again dependent on the height at which the plumes merge. In this case there are three possible flow regimes:

i) The two plumes merge below the lower interface. In this case the single plume model can be used with the interface height given by $\zeta^{\prime}=\zeta_{1}+\varepsilon$ and Equation 12. This is the same as for equal strength plumes, but the merging heights and distance to the virtual origin as functions of separation vary with $\psi$ as shown in Figures 6 and 9 of Kaye and Linden (2004).

ii) The two plumes merge above the height predicted for the lower interface but below the height of the upper interface. The weaker plume is entrained into the stronger plume and only a twolayer stratification will form. Volume and buoyancy flux conservation gives the interface height as:

$\frac{A^{*}}{H^{2}}\left(\frac{1+\psi}{\left(1+\psi^{1 / 3}\right)^{3}}\right)^{1 / 2}=C^{3 / 2}\left(\frac{\zeta_{2}^{5}}{1-\zeta_{2}}\right)^{1 / 2}$

Since the factor:

$\left(\frac{1+\psi}{\left(1+\psi^{1 / 3}\right)^{3}}\right)^{1 / 2} \approx \frac{1}{2}$ for $\psi>0.3$,

the interface will be at approximately the same height as for two equal plumes over a wide range of $\psi$.

iii) The plumes do not interact below the predicted upper layer. The weaker plume will then spread out below the upper interface and never merge with the stronger plume, and the full non-interacting model for unequal co-flowing plumes in a ventilated enclosure can be used. 


\subsection{Experiments}

Experiments were performed to examine the above cases, but physical constraints limited the range of parameter space we could investigate. The experiments were conducted in a Perspex enclosure with a base $40 \mathrm{~cm}$ square. The top of the enclosure was adjustable allowing depths $H$ between $10 \mathrm{~cm}$ and $35 \mathrm{~cm}$. The base and top had 12 holes each, 4 of diameter $5.5 \mathrm{~cm}$ and 8 of $2.0 \mathrm{~cm}$. These holes could be closed or open depending on the desired value of $\mathrm{A}^{*}$. The two plume sources were separated by $2.5 \mathrm{~cm}$.

Turbulent plumes were formed by introducing salt solution through specially designed sources at the top of the ventilated enclosure of known buoyancy at a constant measured flow rate from a constant head tank. The enclosure was immersed in a large tank full of fresh water that acted as the external ambient (Figure 7). The total height $H$ was taken as the distance from the plume source to the base of the enclosure plus the height of the virtual origin of the plume (calculated using the technique of Hunt et al 2001). As the plumes were negatively buoyant the experiment is upside down compared to the previous description. However, all results will be reported as if the plumes were positively buoyant. This reversal in sign is unimportant for the small density differences in these experiments and as experienced in buildings.

The height of the steady-state interface between the ambient upper layer and the dense lower layer was measured. The transient development of these flows is discussed in Kaye and Hunt (2004).

Experimental results are presented for a constant value of $A^{*} / H^{2}$, for different dimensionless separations $\sigma=\chi / H$, by varying the enclosure depth $H$ and adjusting the number of open vents to give the correct dimensionless opening area. The value of $\psi$ was varied between $0.4<\psi<1$. The experimental results for $\sigma=0$ were obtained using a single plume with twice the buoyancy flux.

Figure 8 shows the results for two equal $(\psi=1.0)$ co-flowing plumes with $A^{*} / H^{2}=0.04$, and $\sigma$ varied from 0 to 0.3 . There is excellent agreement between the experimental observations and the theoretical prediction (Equation 12).

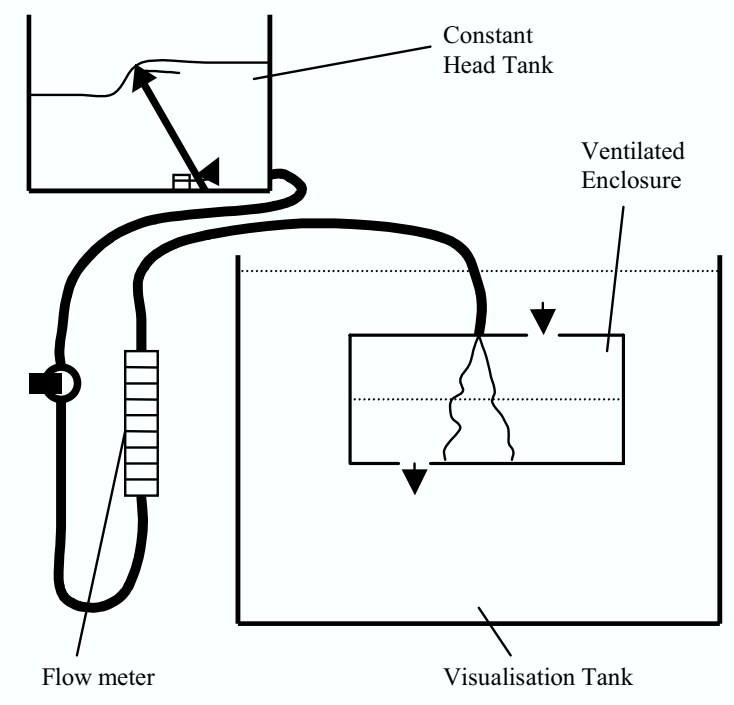

Figure 7. Schematic diagram of the experimental set up.

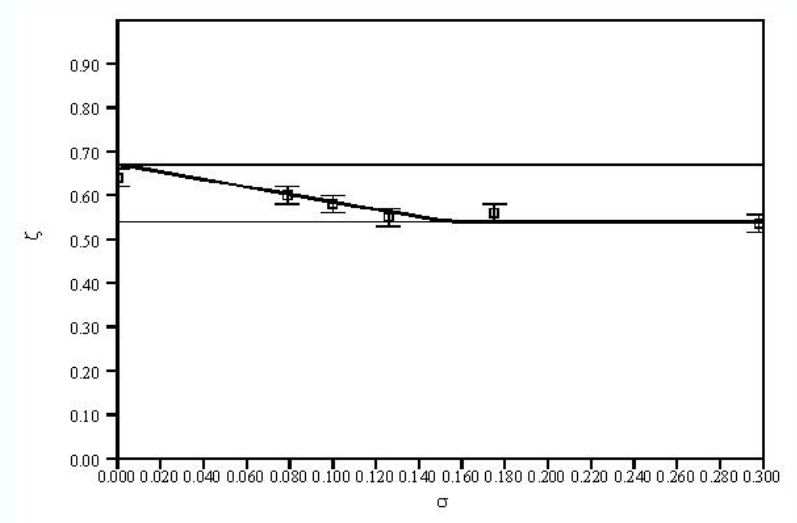

Figure 8. Interface height plotted as a function of the plume separation for two equal plumes with $A^{*} / H^{2}=0.04$. The horizontal lines are the one and two plume solutions, and the thicker line is the theoretical correction for the origin of the merged plume. 


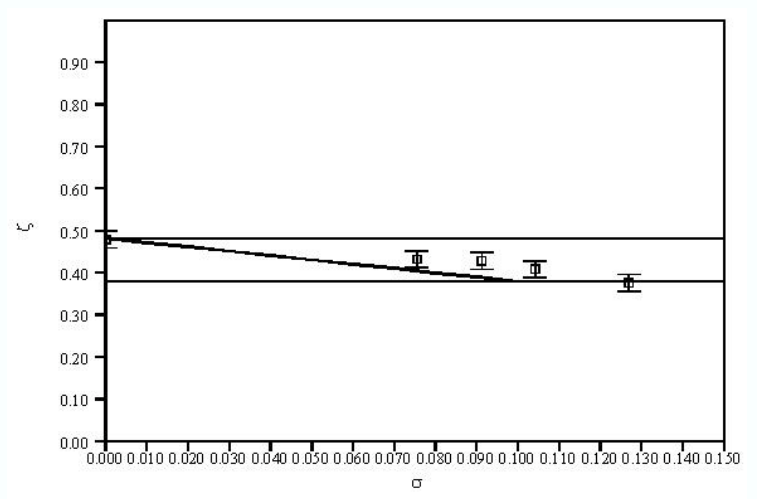

Figure 9. Interface height plotted as a function of the plume separation for $\psi=0.7$ with $A^{*} / H^{2}=0.014$. Theory lines are as for Figure 8.

Figures 9 and 10 show results for unequal plumes with $\psi=0.7$ and 0.4 , respectively, and an effective area of $A^{*} / H^{2}=0.014$. The agreement with the theory is not as good as for the equal plumes, but the predicted approximate linear variation of the interface height with separation between the single plume and two plume solutions is observed.

Figure 11 shows the results for the case where two equal plumes coalesce both above and below the interface. All other parameters were held fixed and only the vent areas were varied to cover a wide range of $A^{*} / H^{2}$.

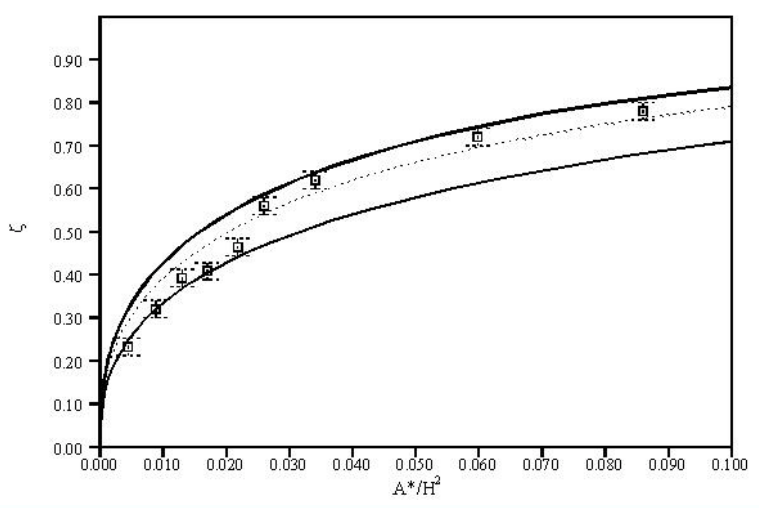

Figure 11. Interface height as a function of area in a ventilated enclosure for $\sigma=0.1$ and $\psi=1.0$. The upper and lower lines are the one-and two-plume solutions and the dashed line is the theoretical correction for the virtual origin of the merged plume.

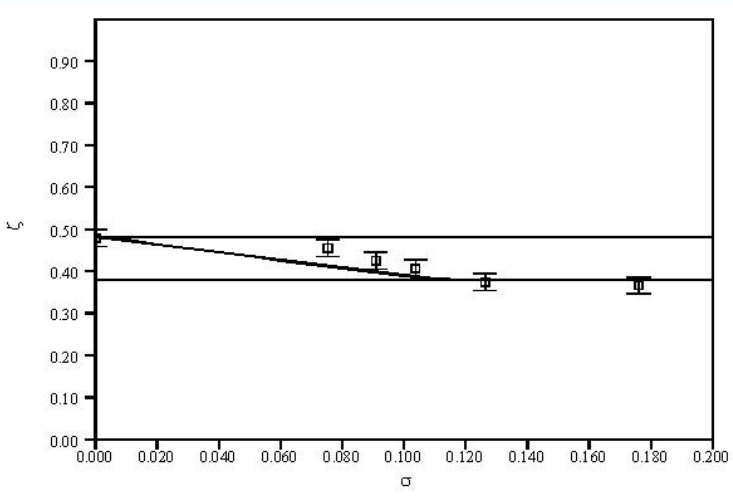

Figure 10. Interface height plotted as a function of the plume separation for $\psi=0.4$ with $A^{*} / H^{2}=0.014$. Theory lines are as for Figure 8.

For $\zeta<\sigma \lambda_{m}=0.48$ the predicted interface height is given by Equation 5. In this parameter range the two plumes were observed to be separate below the interface. Above this height, the predicted interface height is given by Equation 1, with a correction for the virtual origin of the single equivalent plume (Equation 12). This corrected value is shown by the dotted line. The data are in excellent agreement with the theory, and show a sharp jump between the twoplume case and the corrected single plume case around $\zeta \approx 0.5$, as expected.

Due to the constraints of the enclosure geometry the three-layer case was not tested.

\section{Colliding Plumes}

We now examine the problem of counter-flowing turbulent plumes interacting in a ventilated enclosure (Kaye and Linden 2006).

\subsection{Theory}

The purpose of this section is to examine the effect of plume collision on the non-interacting ventilation model of Cooper et al (1996) reviewed above. The opposing plume case has only been examined analytically for small values of $\psi$, where the lower layer is ambient fluid and there is no exchange flow through the enclosure vents. We, therefore, restrict our analysis to the small $\psi$ case, when one plume dominates the other. 


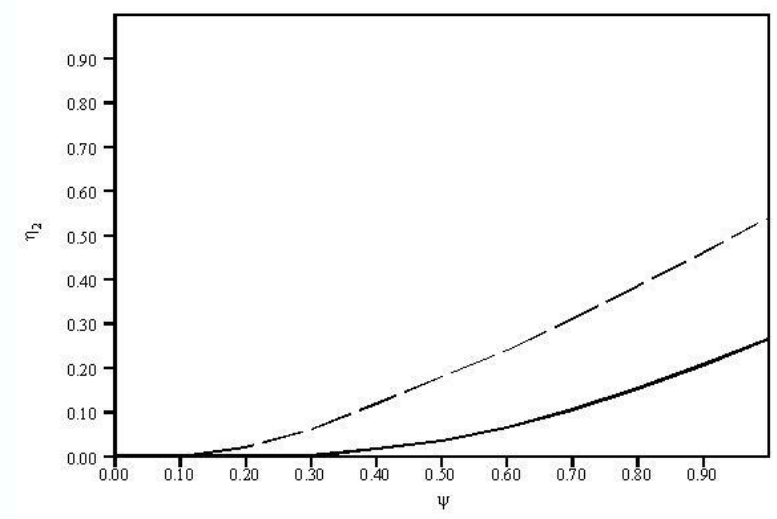

Figure 12. Theoretical prediction for the total loss of buoyancy flux as a function of the $\psi$. The solid line is for $\sigma=0.0$ and the dashed line is for $\sigma=0.09$.

In that case the weaker plume can no longer be treated as separate as it is absorbed by the stronger plume on collision. Figure 12 shows the fraction ( $\left.\eta_{2}=\hat{F}_{2 \text { out }} / \hat{F}_{1 \text { in }}\right)$ of the buoyancy flux of the weaker plume that is not absorbed due to collision with the stronger plume as a function of $\psi$ for plume separations of $\sigma=0.0$ and 0.09 . The "in" and "out" refer to the buoyancy flux of each plume prior to (in) and after (out) colliding with each other. This shows that for plumes aligned vertically $(\sigma=0.0)$ the weaker plume is almost completely absorbed for $\psi<0.4$ as $\hat{F}_{2 \text { out }} \approx 0$. For larger separations the value of $\psi$ at which the plume loses all its buoyancy flux is less.

Balancing the volume flux through the tank with that of the plume at the interface height we get the relationship:

$$
Q=E \hat{F}_{1}^{1 / 3} h^{5 / 3} \quad A^{*} \sqrt{g_{l}^{\prime}\left(\begin{array}{ll}
H & h
\end{array}\right)}
$$

Conservation of buoyancy flux in this case is:

$\left|\hat{F}_{1}\right|-\left|\hat{F}_{2}\right| \quad Q g_{l}^{\prime}$

and, hence, the flow rate is given by:

$$
Q=A^{*}\left(\frac{\hat{F}_{1}-\hat{F}_{2}}{C \hat{F}_{1}^{1 / 3} h^{5 / 3}} \quad(H \quad h)^{\frac{1}{2}} \quad C \hat{F}_{1}^{\frac{1}{3}} h^{\frac{5}{3}}\right.
$$

or in dimensionless form

$$
\frac{A^{*}}{H^{2}}=\epsilon^{3 / 2}\left({\frac{\zeta^{5}}{1-\zeta}}^{1 / 2}(1 \quad \psi)^{-1 / 2}\right.
$$

For $\psi=0$ this leads to the single plume result. For $\psi>0$ we can approximate the solution by following a similar technique to that used in Section 3.

Writing the steady state interface height for a singleplume as $\zeta_{1}$ and the new interface height for two colliding plumes as $\zeta_{1}+\varepsilon$, Equation 19 gives:

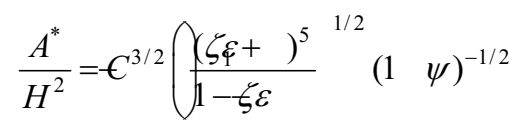

Hence:

$\varepsilon \not=-\bigcap \frac{\zeta_{1}^{5}}{\sqrt[{\frac{A^{*}}{H^{2}}}^{2} C^{3}(1-\psi)]{5 \zeta_{1}^{4}}}$

which is a small correction of order $\psi \zeta_{1} / 5$.

Figure 13 shows the interface height for both the non-interacting and colliding equal-plume cases, for two values of $A^{*} / H^{2}$. The data for the noninteracting case was taken from Cooper and Linden (1996), with a correction to their values as they used a different value for the plume coefficient $\mathrm{C}$.

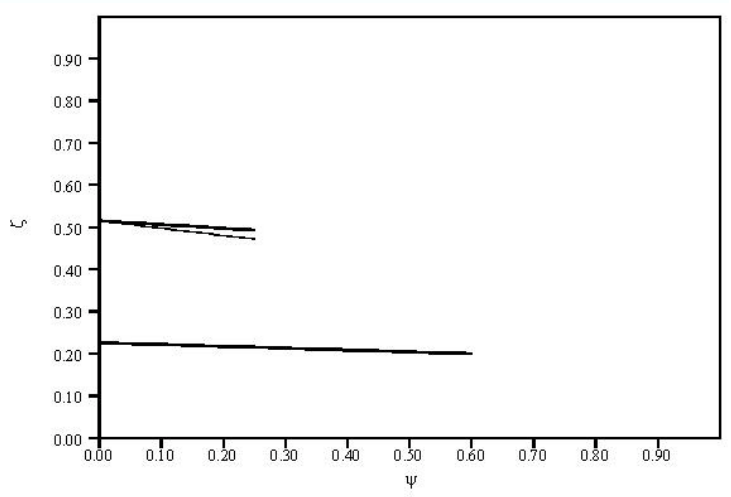

Figure 13. Theoretical prediction of interface height for two colliding plumes. The thin lines are those of Cooper and Linden (1996), and the thick lines are a correction allowing for the absorption of the weaker plume into the stronger. The top pair of lines are for

$A^{*} /\left(H^{2} C^{3 / 2}\right)=0.0174$ and the lower pair for

$A^{*} /\left(H^{2} C^{3 / 2}\right)=0.00174$. Note that for the lower set only the thick line shows as there is a negligible difference between the curves. 


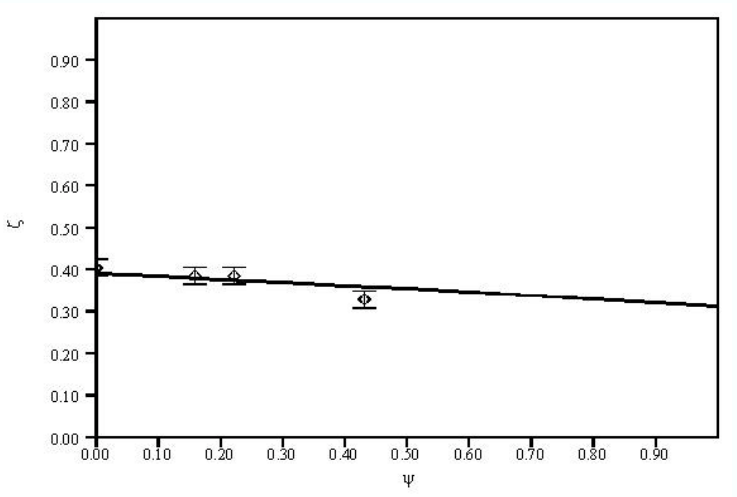

Figure 14. Interface height plotted as a function of buoyancy flux ratio with $A^{*} / H^{2}=0.0078$ and $\sigma=0.0$.

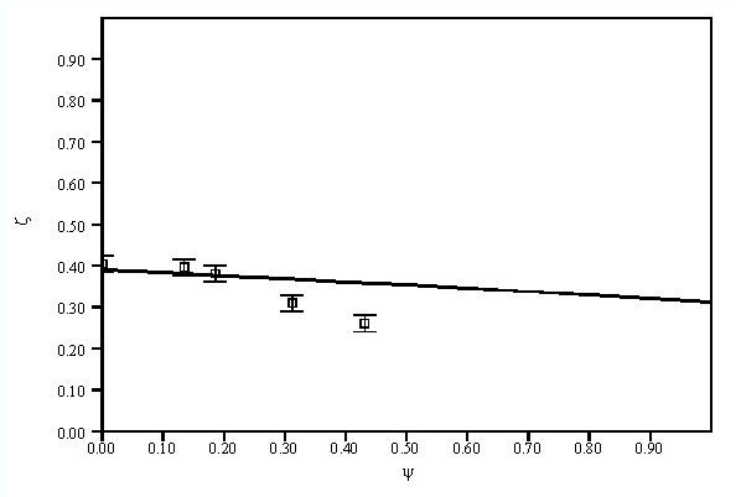

Figure 15. Interface height plotted as a function of buoyancy flux ratio with $A^{*} / H^{2}=0.0078$ and $\sigma=0.9$.

\section{Conclusions}

figure shows that, for the larger opening area, the effect of the plume interaction is to raise the interface, mainly because the weaker plume is absorbed. Consequently it does not impinge on, or entrain fluid across, the interface. This difference is reduced for the smaller opening area. In that case the rate of entrainment across the interface for the non-interacting case is less, as the weaker plume has to travel further through the positively buoyant layer before it reaches the lower interface. In any case the effects of collision on the interface height are small, since it is set by the entrainment into the separate plumes before they collide.

\subsection{Experiments}

Experiments were performed for two values of $\sigma$ over a range of $\psi$ to test the validity of the model. The negatively buoyant plume was a salt solution as before and the positively buoyant plume was a $25 \%$ by volume mixture of Propan-2-ol and water with a reduced gravity of $g^{\prime} \approx 30 \mathrm{cms}^{-2}$.

Experiments were conducted for $\sigma=0.0$ and 0.09 , with $A^{*} /\left(H^{2} C^{3 / 2}\right)=0.0078$. The results (Figures 14 and 15) show that the interface height varies only slightly with $\psi$ in the range where the weaker plume is expected to be absorbed. Outside that range, the weaker plume was observed to impinge on the lower interface causing the interface to be lower than predicted by the model. This departure from the theoretical curve occurs for a lower value of $\psi$ for $\sigma=0.09$ than for $\sigma=0.0$, and is expected as the theory only applies when the weaker plume is completely absorbed $\left(\eta_{2} \approx 0\right.$ - Figure 12$)$.
We have considered the effects of the interaction of two co-flowing and two counter-flowing plumes in a ventilated enclosure that is in steady displacement ventilation. When the plumes are close together their mutual entrainment causes them to converge and combine into a single plume. The coalescence of co-flowing plumes only affects the stratification if they coalesce below the interface height expected for two separated plumes. In that case the new steady state interface height can be predicted by assuming that there is only a single combined plume, with increased buoyancy flux and a virtual origin (Equation 12). If the coalescence occurs above the interface, its height is given by noninteracting theory.

Kaye and Linden (2004) showed that two plumes of similar strength coalesce at a height of between 4 and 5 times the horizontal separation of their sources. For a typical ventilated office with ceiling height $2.5 \mathrm{~m}$ and an interface height at $1.5 \mathrm{~m}$, plume-plume interaction is significant only if the plume source separation is less than $0.4 \mathrm{~m}$. In practical terms this is very close, and if the dominant heat sources were people then it is unlikely that they would be that close together. In a larger room, such as a lecture theatre, the ceiling could be, say, $8 \mathrm{~m}$ high with the interface at about $4-5 \mathrm{~m}$. In this case people would only have to be sitting around $1 \mathrm{~m}$ apart, wider than a typical chair. Consequently, the interaction of co-flowing plumes can be important in a tall, high occupancy, naturally ventilated enclosure. 
Colliding plumes often have much smaller radial separations as the buoyancy sources are also vertically separated. For example, it is possible to place an air-conditioning vent directly above a computer terminal. However, plume-plume collision will not occur beyond a separation to height ratio of $\sigma \approx 0.2$. For small values of $\psi(<0.3)$ and $\sigma(<0.1)$ it is possible to predict the behaviour of the steady state stratification by assuming that the weaker plume is absorbed by the stronger plume. The interface height is given by balancing the flow rate in the stronger plume at the interface, with the flow driven through the enclosure by the upper layer. The upper layer buoyancy is the net buoyancy flux $\left(\left|\hat{F}_{1}\right|-\left|\hat{F}_{2}\right|\right)$ divided by the stronger plume flow rate at the interface height.

Although the adjustments to the ventilation model presented above do expand the parameter range over which ventilation problems can be solved, it is still far from a complete model. This work demonstrates the need to model heat source conditions accurately, in order to predict how well a ventilation system will operate.

\section{References}

Baines WD and Turner JS: (1969) "Turbulent buoyant convection from a source in a confined region". J. Fluid Mech., 37, pp51-80.
Cooper P and Linden PF: (1996) "Natural ventilation of an enclosure containing two buoyancy sources". J. Fluid Mech., 311, pp153-176.

Gladstone C and Woods AW: (2001) "On buoyancy-driven natural ventilation of a room with a heated floor". J. Fluid Mech., 441, pp293-314.

Kaye NB and Linden PF: (2004) "Coalescing axisymmetric turbulent plumes". J. Fluid Mech., 502, pp41-63.

Kaye NB and Linden PF: (2006) "Colliding turbulent plumes”. J. Fluid Mech., 550, pp85-109.

Kaye NB and Hunt GR: (2004) "Time dependent flows in an emptying filling box". J. Fluid Mech., 520, pp135-156.

Linden PF and Cooper P: (1996) "Multiple sources of buoyancy in a naturally ventilated enclosure". $J$. Fluid Mech., 311, pp177-192.

Linden PF, Lane-Serff GF and Smeed DA: (1990) "Emptying filling boxes, the fluid mechanics of natural ventilation”. J. Fluid Mech. 212, pp309-315.

Hunt GR and Kaye NG: (2001) "Virtual origin correction for lazy turbulent plumes". J. Fluid Mech., 435, pp377-396. 